\title{
Optimization Research on Process of Airport Security Check
}

\author{
Huayanran Zhou ${ }^{1, \text { a }}$ \\ ${ }^{1}$ School of North China Electric Power University, Hebei 071000, China \\ a364876093@qq.com
}

Keywords: Petri net, Air transportation, Markov chain, Security check process optimization.

\begin{abstract}
To reduce the waiting time for safety check in airports, and improve the transport efficiency, the safety checking model should optimized based on the premise of safety. Since security system has a concurrency, and Petri net can express concurrent events, Petri net model is used to solve this problem. After analyzing the average number of token utilization and change, Millimeter Wave Scan times and Time to get scanned property are chosen as potential modifications to the current process which can improve passenger throughput and reduce variance in waiting time. Then, apply GSPN and Markov Chain theories to evaluate the performance of security check process. Finally, take Chicago O'Hare Airport as an example, put forward suggestions and design the security process improvement.
\end{abstract}

\section{Introduction}

The current way of airport security check requires a couple of seconds to complete the whole inspection process which has largely led to the inconvenience and inefficiency for passengers. In 2016, the TSA, especially O'Hare International Airport, received an increasing number of complaints and criticisms for the long for security check. To address this problem, the TSA has invested in modifying the inspection devices and checking procedures, as well as increasing the number of airport staff at highly congested airports. However, these long waiting problems still exist. To reduce the waiting time for safety check in airports, and improve the transport efficiency, the safety checking model should optimized based on the premise of safety.

In this paper, we study on the flow of aviation passengers passing through a security check point and its bottlenecks, therefore identify the main points where problems exist in the current process.Then, modifying the current process to improve the throughput efficiency of aviation passengers and reduce variance in waiting time. Meanwhile, the modification is demonstrated to optimize the process.

\section{Model Building}

\section{Single Channel Security Process Modeling.}

Petri net is an effective modeling tool for describing and analyzing concurrent and synchronous feature systems. As a system, the process of passenger security inspection has the characteristics of synchronization and concurrency. Besides, according to the actual situation of the security system, the time parameter is introduced to analyze the time behavior of the model. We choose the generalized stochastic Petri net model (GSPN) to model the passenger security process.

According to the definition of Petri net, combined with the terminal passenger screening process, we establish a simple single channel screening process Petri net model --- there is only one authentication table, a detector, a X machine, a hand inspector. As shown in the Fig. 1. 


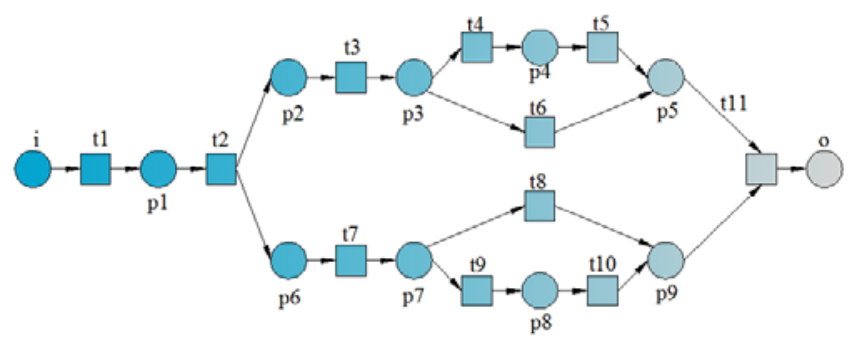

Fig. 1 Petri network model for passenger security checkpoint process

\section{Model Performance Analysis.}

A stochastic Petri net model is equivalent to a continuous time Markov Chain. The reachability graph can be obtained by the established GSPN model, and a Markov Chain with the constructed GSPN model is obtained after processing. Markov Chain is used to solve the steady state probability, and then the marking probability density, the average number of markers in the library, the utilization rate of change, etc. Utilizing the results, the performance index of the system security analysis will first find the bottleneck in the screening process, and then sort out a breakthrough in optimizing the system, change the security facilities, reasonable arrangement of security resources, and finally shorten the screening process of Business Hours.

In order to ensure that GSPN is a continuous process and maintain its strong connectivity, we add temporal change $t$ and its initiation rate $(\lambda)$. When then set up a list of reachable flags and reachable flags.Second, construct the Markov Chain with the GSPN Model, and establish the density matrix. Third solve the Steady State Probability of Markov Chain. The probability of each state is $\mathrm{X}=(\mathrm{x} 0$, $\mathrm{x} 1, \ldots, \mathrm{x} 18)$, using the equation $\mathrm{X} \cdot \mathrm{Q}=\mathrm{O}$ and combining it with the density matrix $\mathrm{Q}$, we obtain the probability distribution of the steady state. Finally, according to the probability of the steady state distribution determined by the security system in every place the average token number and the change rate, analysis of performance indicators of the security system, and find the bottleneck of security system, and finally put forward the optimization scheme.

Table 1 The average number of token utilization change change

\begin{tabular}{|c|c|c|c|}
\hline The libraries & The average number of token & change & Utilization rate of change \\
\hline $\mathrm{I}$ & - & $\mathrm{t} 1$ & 0.123640 \\
\hline $\mathrm{p} 1$ & 0.09711 & $\mathrm{t} 3$ & 0.067460 \\
\hline $\mathrm{p} 2$ & - & $\mathrm{t} 7$ & 0.162064 \\
\hline p3 & 0.16445 & $\mathrm{t} 11$ & 0.341643 \\
\hline p4 & - & & \\
\hline p5 & - & & \\
\hline p6 & 0.10314 & & \\
\hline p7 & - & & \\
\hline p8 & - & & \\
\hline p9 & 0.39416 & & \\
\hline O & - & & \\
\hline
\end{tabular}

As can be seen from table 1, P9 and P3 has large the number of tokens, so these two places are easy to form the bottleneck. The number of tokens of P6 is also relatively large. P3 is the baggage checked by X-ray machine, while P9 is the part of passengers picking up their luggage and P6 is Millimeter wave scan. A possible reason is that the baggage extraction process is longer by passengers.

The utilization ratio of $\mathrm{t} 11$ is high. We analyze that there is a shortage of hand-held detector inspectors and a longer time for passengers to take out luggage. 


\section{Optimization}

\section{Suggestions.}

Taking Chicago O'Hare Airport as an example, the main bottleneck exists in the screening process, personal check and luggage unpacking inspection link. The following suggestions are proposed.

Standardized operation level. Establish a sound operational norms, strengthen the training of security personnel and improve the level of operation of security personnel.

Increase the number of staff. Due to the fast speed of the door detection and relatively slow the staff detection, passengers are crowned in the field of physical examination. So we can increase the number of hand inspectors to catch up with the speed of the security door.

Increase the number of X-ray machines or set up special baggage sorting areas. In order to reduce the influence of the long time-period of baggage extraction on the efficiency of security inspection, on the one hand, we can increase the number of X-ray machines, which can decreasing the amount of baggage in front of each machine, on the other hand, we set up a dedicated platform in the X-ray machine exit and arrange personnel to help passengers sort their baggage. Although the increase in the number of X-ray machines can alleviate congestion as well as improve efficiency, the use of $\mathrm{X}$-ray light opportunities would also lead to high costs. Therefore, the problems can be solved through increasing the X-ray machine number as well as setting up platforms especially for inspecting and sorting out baggage.

\section{Optimized Data Analysis.}

In order to facilitate the study, we assume that the probability of each passenger to choose the same security service resources are equal, and each increase in a certain class of security services resources would correspondingly double the change of the growth rate. Statistics show that personal inspection service time varies, so we assume that the service time after training can be reduced to $6 \mathrm{~s}$; and the period can be shortened to $8 \mathrm{~s}$ after the establishment of the special baggage inspection and sorting platform.

Table 2 The average number of token optimized library

\begin{tabular}{|c|c|c|c|}
\hline $\begin{array}{c}\text { The } \\
\text { libraries }\end{array}$ & The average number of token & $\begin{array}{c}\text { The } \\
\text { libraries }\end{array}$ & The average number of token \\
\hline $\mathrm{i}$ & - & $\mathrm{p} 5$ & - \\
\hline $\mathrm{p} 1$ & 0.08974 & $\mathrm{p} 6$ & 0.10131 \\
\hline $\mathrm{p} 2$ & - & $\mathrm{p} 7$ & - \\
\hline $\mathrm{p} 3$ & 0.12134 & $\mathrm{p} 8$ & - \\
\hline $\mathrm{p} 4$ & - & $\mathrm{p} 9$ & 0.19634 \\
\hline
\end{tabular}

Due to the increase of X-ray machine, tokens shunting. The token of P3( baggage checked by $\mathrm{X}$-ray machine),P9(passengers preparing for catching the luggage) reduce obviously. It is proved that the optimization scheme can effectively deal with the bottleneck and improve the efficiency of security inspection.

\section{Conclusions}

In a security channel set an identity verifiel, a security detection gate, two X-ray machine, two workers. Simple diagram as shown in Fig. 2. 


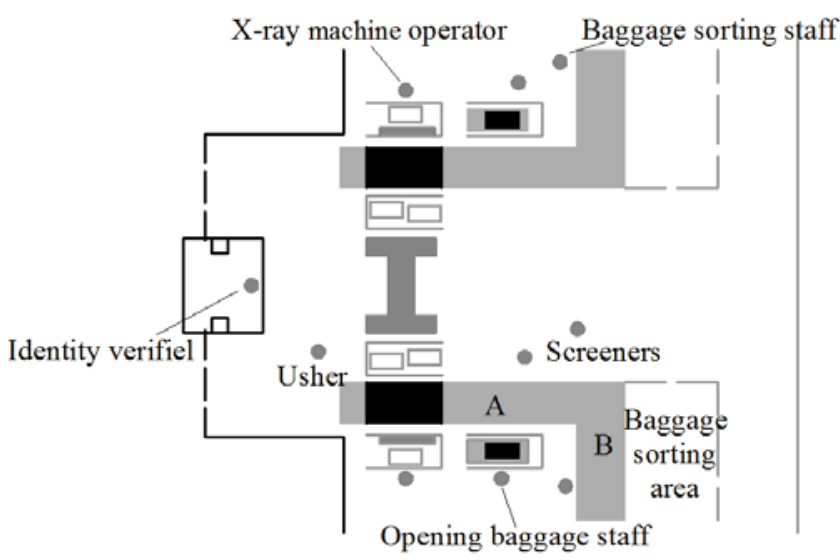

Fig. 2. Security process improvement design

After authentication, passengers enter the security area, selecting a free X-ray machine to check their luggage, and then go through the hassles. If the security alarm rings, passengers will choose a free staff to do personal checks. If there is any suspicious item in luggage, passenger will go to Zone $\mathrm{A}$, opening their luggage for check. If there is no problems, passengers can take their luggage at Zone B. While passengers are under the personal check, staff in Zone B can arrange the luggage so that their baggage in Zone B once they finish their personal check. In terms of the specific layout of the security check area at the terminals, it can be adjusted according to the actual situations.

\section{References}

[1] Sweet K. Aviation and Airport Security: Terrorism and Safety Concerns[M]. Florida: CRC Press, 2011.

[2] Van Der Aalst W M P. Workflow Verification: Finding Control-flow Errors using Petri - net based Techniques [M]. Heidelberg, Berlin: Springer,2000.

[3] David R, Alla H. Discrete, Continuous, and Hybrid Petri Net [M] . Heidelberg, Berlin: Springer Science \& Business Media, 2010.

[4] Wang Y, Lin C, Ungsunan P D,et al. Modeling and Survivability Analysis of Service Composition using Stochastic Petri Nets $[\mathrm{J}]$. The Journal of Supercomputing,2011, 56( 1) : 79105. 